\title{
Aortic valve neocuspidization with glutaraldehyde-treated autologous pericardium to avoid the prosthesis-patient mismatch of a severely obese 57-year-old patient-a case report
}

\author{
Alberto Albertini, Eliana Raviola, Fabio Zucchetta, Carlotta Brega, Elisa Mikus, Alberto Tripodi \\ Cardiovascular Surgery Unit, Maria Cecilia Hospital GVM Care \& Research, Cotignola, Italy \\ Correspondence to: Alberto Albertini, MD. Cardiovascular Surgery Unit, Maria Cecilia Hospital GVM Care \& Research, Cotignola, Italy. \\ Email: aalbertini@gvmnet.it.
}

\begin{abstract}
Aortic valve repair is the gold standard for aortic valve diseases and it is mandatory, every time it is possible, to spare the valve, especially in adults. In non-repairable aortic valves, the most common treatment is replacement with valve prostheses. Despite the risk of reoperation, bioprosthesis are often implanted in non-elderly adults. The durability of the bioprostheses in youngers is lower than in the elderlies. Immune reaction, shear stress and calcium metabolism can play a fundamental role in degeneration. The alternative to prosthetic aortic valve replacement is the Ross procedure, which apparently is the best option for this patient, but its complexity combined with the need for intervention on both aortic and pulmonary valves have limited the use of this technique over time. A more recent alternative is aortic valve neocuspidization, as outlined by Ozaki, which consists in aortic valve reconstruction using glutaraldehyde treated autologous pericardium. Seen the recent positive outcomes reported with this technique, we decided to submit the patient, an obese 57 years old female affected by severe rheumatic aortic valve stenosis and moderate mitral valve stenosis, to this procedure. Our idea was to reduce the risk of prosthesis-patient mismatch (PPM) that this patient faced, seen that she had a small aortic annulus, but a high body mass index. Results have been great: aortic valve gradients were low at discharge and at follow up. Learning from this case, we can assert that this kind of surgery can be safely performed achieving superior hemodynamic results and quality of life. Thus, we did not perform many cases of Ozaki neocuspidization, we always looked for the "perfect patient": young, without big comorbidities, thin. The case we describe obliged us to face with a problem that today's surgeons find out more and more in their clinical practice: obesity in young adults. It is a matter of fact that when a prosthetic valve is implanted the risk of PPM is always behind the corner for the valve structure itself and the big amount of prosthetic material it is composed by. Performing echocardiography and angio CT scan in patients who underwent aortic valve neocuspidization the effective orifice of the valve and the transvalvular gradients where always lower than in those patients who underwent replacement with a prosthesis. This fact has been the starting point for the thought that, maybe, Ozaki technique is the best solution for obese patients and this case proved us right.
\end{abstract}

Keywords: Aortic valve replacement; aortic valve neocuspidization; autologous pericardium; nonelderly adults; case report

Received: 09 April 2020; Accepted: 11 August 2020; Published: 20 July 2022.

doi: $10.21037 /$ jovs-20-110

View this article at: http://dx.doi.org/10.21037/jovs-20-110 


\section{Introduction}

Every year $20 \%$ of patients who undergo aortic valve replacement surgery (AVR) are under 60 years of age (1). In this category of patients, the best therapeutic choice in terms of technique and aortic valve prosthesis presents a controversial issue. The younger the patient, the greater the risk that his life expectancy will be linked to problems caused by valve-related complications and for reoperation.

The literature proves that Ross procedure is the best option for this patient in opposition to valve replacement with mechanical or biological prosthesis (2). Despite this, however, this technique is used in less than $3 \%$ of cases and is not laid out in the latest European guidelines, while in the U.S. ones $(3,4)$ its use is considered only for patients who have a contraindication to anticoagulation. The limited use of the Ross technique is due to the great complexity and high incidence of reoperation on both the aortic valve and pulmonary conduit, so in the real world the majority of the aortic valves are replaced with prostheses.

As an alternative to the Ross procedure, Ozaki has described (5) a new technique of aortic valve reconstruction with the use of autologous pericardium treated with glutaraldehyde, called aortic valve neocuspidization. This technique has given satisfactory results in young adults in terms of mid-term durability and is very effective in reducing the rate of valve-related complications. The idea of using autologous tissue in order to avoid anticoagulation represents one of the main advantages of this procedure, thus it is performed on the aortic valve alone, unlike the Ross procedure. Furthermore, the absence of synthetic tissue such as valve stent, sewing ring or cuff reduces the overall risk of infective endocarditis.

Our centre has already performed more than 40 Ozaki procedures. While the first cases have been done on those defined "good patients" (few comorbidities, thin, young), the experience we have matured moved us towards new challenges. One of these are obese patients, a growing reality in our practice. Imaging techniques (CT scan, echocardiography) show that this procedure allow us to optimize the space, creating a valve that has almost the same surface of the aortic annulus and, according to the law of Poiseuille, let a big area for the blood to flow. That is the reason why aortic valves replaced with Ozaki technique have lower transvalvular gradients and flow velocity if compared to prosthesis, in every kind of patient. This is very useful when we approach a patient with a high body surface, but a small annulus, exactly like the case we write about in this article. We present the following case in accordance with the CARE reporting checklist (available at https://jovs. amegroups.com/article/view/10.21037/jovs-20-110/rc).

\section{Case presentation}

We present the case of a 57-year-old woman with history of acute articular rheumatism in childhood. During the last month before the operation the patient began rapidly symptomatic, showing signs of dyspnoea and angina following moderate physical exertion (NYHA class II).

The patient was examined at the Cardiology Outpatient Clinic using a transthoracic echocardiogram (TTE), that revealed a severe aortic stenosis with an area of $0.7 \mathrm{~cm}^{2}$, a maximum gradient of $113 \mathrm{mmHg}$ and a mean gradient of $67 \mathrm{mmHg}$ and a moderate mitral valve stenosis with an area of $1.5 \mathrm{~cm}^{2}$ and a mean gradient of $10 \mathrm{mmHg}$. The systolic pulmonary artery pressure was $65 \mathrm{mmHg}$ and the left ventricle had preserved ejection fraction with severe hypertrophy. The patient was admitted to our cardiovascular surgery division where he underwent a transoesophageal echocardiogram (TEE) that confirmed the diagnosis and a coronary artery computed tomography (CT) angiogram with no evidence of coronary arteries stenosis.

The case was discussed by our local Heart Team and surgical indication for aortic valve replacement and debridement of the mitral valve was given. Despite the patient was severely obese (BMI $\left.42 \mathrm{~kg} / \mathrm{m}^{2}\right)$, the diameter of aortic annulus, measured through CT scan and TEE, was only $23 \mathrm{~mm}$. These parameters indicated that, regardless of the type of prosthesis that would have been implanted, the patient would have met a prosthesis-patient mismatch (PPM); in order to avoid this complication, we decided to proceed with an aortic valve neocuspidization as this technique ensures better haemodynamic performance in terms of postoperative aortic transvalvular gradients and, furthermore, significantly lowers the risk of PPM.

All procedures performed in this study were in accordance with the ethical standards of the institutional and/or national research committee(s) and with the Helsinki Declaration (as revised in 2013). Written informed consent was obtained from the patient for publication of this case report and accompanying images. A copy of the written consent is available for review by the editorial office of this journal.

\section{Surgical procedure}

The intervention was performed under general anaesthesia, 


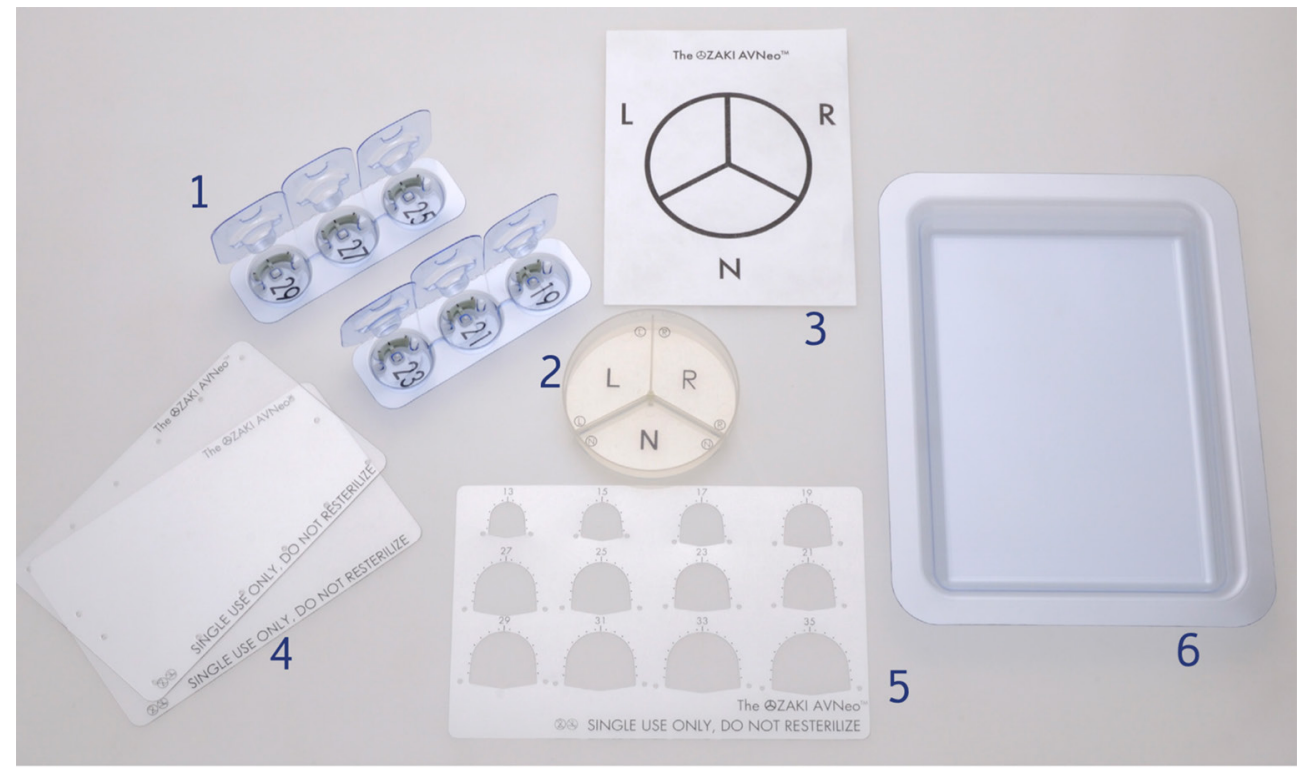

Figure 1 The AVNeo ${ }^{\mathrm{TM}}$ kit. 1: AVNeo standard sizers; 2: orientation dish; 3: leaflet-size identification sheet; 4: pericardium fixation plates; 5: leaflet tracing templates; 6: glutaraldehyde tray.

through a complete median sternotomy and the whole procedure was carried out using the Ozaki-AVNeo kit, containing special instruments (JOMDD Inc., Tokyo, Japan) (Figure 1).

A $7 \mathrm{~cm} \times 8 \mathrm{~cm}$ long sheet of anterior autologous pericardium was harvested using surgical scissors and then stretched by fixing it onto a special plastic plate using 16 stitches of $2 / 0$ polyethylene terephthalate. Surgical debridement of the pericardium was performed to remove fat and redundant tissue on the outer surface and then soaked in a $0.6 \%$ glutaraldehyde solution for 10 minutes. Once removed from the plaque, it was rinsed in three different cups filled with $500 \mathrm{cc}$ of physiological saline solution for 6 minutes each. During this interval, cardiopulmonary bypass was established and the heart arrested by infusing a single dose of cold crystalloid Custodiol cardioplegia in the aortic root. A transverse aortotomy was performed about $5 \mathrm{~mm}$ above the commissures and the aortic valve has been exposed; it presented severe calcifications of the leaflets and the annulus. The diseased cusps were excised and the annulus have been debrided from the calcium, including the calcium build-up rising from the aortic annulus resulting in mitral stenosis by impairing the movement of the anterior leaflet. The aortic annulus was exposed with the help of two Herc-Flex stabilizer arms (Terumo Inc., Tokyo, Japan), each Valsalva sinus was measured calculating the distance between one commissure and the next using the specific sizing system of the Ozaki-AVNeo kit. During the sizing, it is fundamental to maintain the right tension on the annulus in order to reproduce the diastolic phase of the cardiac cycle and every Valsalva sinus nadir was marked. Sizing is crucial in this technique as three completely new aortic cusps are created reproducing the native anatomy of the aortic valve and an error could lead to the failure of the entire procedure. Measurements were noted on a specific schematic paper sheet contained in the kit. The pericardium was posed on a second plate with the smooth surface upward, the cusps were drawn with the surgical marker according to the corresponding size window on the template and the dots driving the sewing line were drawn at $1.5 \mathrm{~mm}$ from the edge of the cusp, then the pericardium was trimmed with scissors along the outside edge of the lines and $5 \mathrm{~mm}$ of lateral wing extensions were made at both sides of the cusps. The cusps were placed in the specific tray divided the corresponding Valsalva sinus. Cusps were sutured following the dots to the annulus from downward with a $13 \mathrm{~mm}$ needle- $90 \mathrm{~cm}$ 4/O polypropylene with the smooth surface facing the ventricular side, sutures run from the midpoint of each Valsalva sinus and reach the same horizontal plane as the commissures. suturing the pericardium must be laid underneath the annulus. 


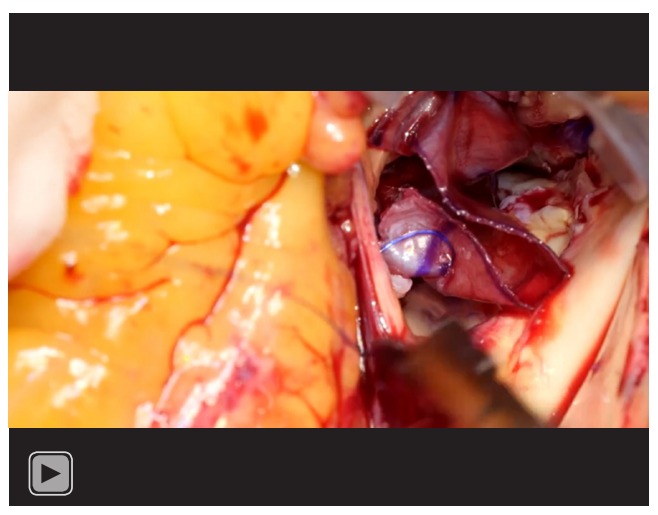

Video 1 Aortic valve neocuspidization with glutaraldehyde-treated autologous pericardium in a severely obese non-elderly patient: a way to avoid the prosthesis-patient mismatch.

The end of the suture was passed through the aorta about $2 \mathrm{~mm}$ below the commissure, the commissural coaptation was then secured with an additional stich of a $17 \mathrm{~mm}$ needle- $90 \mathrm{~cm} \mathrm{4/0}$ polypropylene suture, the needle was passed $2.5 \mathrm{~mm}$ from the edge of both cusps and then penetrated the wings on each side and it was subsequently passed through the aortic wall. The suture and the stitch were then passed on different planes in the same pledget outside the aorta and tied. Once all the cusps were sewn and fixed, the coaptation was ensured with a visual check under negative pressure made by the left ventricular vent. The aortotomy was closed and the heart restarted, weaning from cardiopulmonary bypass (CPBP) was regular.

Intraoperative TEE showed the reconstructed aortic valve without regurgitation, with a mean gradient of $8 \mathrm{mmHg}$ and with an indexed effective aortic orifice area (iEOA) of $0.85 \mathrm{~cm}^{2} / \mathrm{m}^{2}$ (Video 1).

The postoperative period was uneventful and the patient was discharged from hospital on the $8^{\text {th }}$ postoperative day.

A follow-up visit has been performed after 7 months: the patient was asymptomatic for dyspnoea and was on sinus rhythm. TTE showed a preserved ejection fraction (60\%) and a well performing aortic valve with a medium gradient of $12 \mathrm{mmHg}$ and with trivial central regurgitation.

\section{Discussion}

In young adults AVR is the most widely used treatment for aortic valve diseases. Two types of valve substitutes are available for AVR: mechanical and biological prosthesis.

In the last European and American guidelines the use of mechanical valves is recommended in patients younger than 60 and 65 years respectively because of the lower, albeit not null, risk of reoperation compared to the bioprostheses. In both cases, mechanical valves require life-long anticoagulation therapy with a moderate risk for thromboembolic and haemorrhagic events and a general impairment to quality of life. In the real world bioprosthetic valves are increasingly being used for the treatment of non-elderly patients because of freedom from anticoagulation and better quality of life. It is always true, however, that biological prosthesis has limited durability due to deterioration and outcomes reported in literature are worse than those for mechanical valves in terms of need for reoperation and life expectancy (6).

The third option is the Ross operation, described by Donald Ross in 1967 (7), which provides many benefits over the other aortic valve substitutes, namely: lower thrombogenicity, lower risk of endocarditis, better hemodynamic performance, freedom from anticoagulation and an overall increased life expectancy. Despite these excellent results and a long history of improvement to patients' lives, this technique is underused: in fact, it is only applied in less than $3 \%$ of the patients because of the overall complexity of the procedure and of the involvement of both aortic and pulmonary valves. This is a point to consider because the operation could turn a single valve in a potential double valve disease due to the degeneration of the pulmonary autograft in the aortic position and of the allograft in the right ventricle outflow tract (RVOT), an eventuality that occur in all the patients operated before the $18^{\text {th }}$ year of age. This incidence tends to decrease with age, however a significant percentage of patients' needs to have either one or both valves re-operated and the lifetime risk of reintervention remains high. Another limitation to the Ross procedure is the availability of pulmonary allografts, as in many countries homograft banks do not exist or the use of cadaver tissues is forbidden for ethical or religious reasons.

Physicians and engineers constantly look for new solutions for AVR, trying to create new bioprosthesis characterized by long durability that can be implanted in younger patients. In 2011, Chan et al. (8) described a stentless aortic valve model build with autologous pericardium treated with glutaraldehyde tested in a small series of 11 patients with good mid-term results and freedom from structural valve degeneration (SVD) of $100 \%$ at 7.5 years.

More recently S. Ozaki has described in detail a new reconstruction technique of the aortic valve by creating new 
cusps with glutaraldehyde treated autologous pericardium: the aortic valve neocuspidization. Ozaki (9) has also reported a wide series of 1,100 patients treated, with a freedom from reoperation at 12 years of $95.8 \%$. It must be pointed out that in Ozaki's series the majority of reoperations were not related to SVD but to infective endocarditis or technical issues, such as leaflet rupture or recurrent aortic valve regurgitation.

With aortic valve neocuspidization, incidence of valve related complications such as thromboembolism, haemorrhage, infective endocarditis and life expectancy are comparable to those reported for the Ross operation, but incidence of reoperation for SVD is far lower. The concept of using autologous pericardium in aortic valve neocuspidization can be considered the main similarity to Ross procedure, in which the autologous pulmonary valve is used to replace the aortic one, but with the need to replace the RVOT with an allograft. The immune response between the host and the implanted tissue can play a role in the complex mechanisms of valve bioprostheses degeneration, furthermore the shear stress on the leaflets caused by the residual transvalvular gradients in biological AVR also contribute to SVD. The minor activation of the immune system combined with the better hemodynamic performance, which results in less shear stress, can explain the excellent results reported by Ozaki.

On the other hand, even if results are consistent, this is one single center and one single surgeon's experience and up to now these results have not been reproduced by others. Only a few other series are reported with good mid-term results on the whole.

The aortic valve neocuspidization is a promising alternative technique for AVR and results from Ozaki's experience are outstanding in terms of valve related complications and freedom from reoperation. However, such good results in big populations must be confirmed by wider studies with firm results from various centres.

\section{Conclusions}

The aortic valve neocuspidization is a safe and feasible technique and nowadays represents the fourth option for non-elderly patients in alternative to biological or mechanical AVR or Ross procedure.

The minimal immune activation due to the fact that the neo-cusps are implanted directly on the native aortic root and the preserved physiological excursions of the aortic annulus are probably the elements that play a fundamental role in the durability of the reconstructed aortic valve. Specific training is advisable in order to be able to perform this technique properly, as every single step must be known down to the smallest detail. Aortic valve neocuspidization is a procedure of high complexity, that grows exponentially when the native valve is mono or bicuspid.

The promising results reported by Ozaki have to be confirmed, as the overall experience with autologous pericardium is limited, and larger studies involving a bigger number of patients and randomized trials comparing the results with bioprosthetic valves are needed.

\section{Acknowledgments}

Funding: None.

\section{Footnote}

Provenance and Peer Review: This article was commissioned by the Guest Editors (Roberto Di Bartolomeo, Davide Pacini, Mohamad Bashir) for the series "Best Video Presentation Prize for the 10th Postgraduate Course on 'Surgery of the Thoracic Aorta' in Bologna" published in fournal of Visualized Surgery. The article has undergone external peer review.

Reporting Checklist: The authors have completed the CARE reporting checklist. Available at https://jovs.amegroups. com/article/view/10.21037/jovs-20-110/rc

Conflicts of Interest: All authors have completed the ICMJE uniform disclosure form (available at https://jovs. amegroups.com/article/view/10.21037/jovs-20-110/coif). The series "Best Video Presentation Prize for the 10th Postgraduate Course on 'Surgery of the Thoracic Aorta' in Bologna" was commissioned by the editorial office without any funding or sponsorship. AA reports personal fees from JOMDD Inc., outside the submitted work. The authors have no other conflicts of interest to declare.

Ethical Statement: The authors are accountable for all aspects of the work ensuring that questions related to the accuracy and integrity part of the work are appropriately investigated and resolved. All procedures performed in this study were in accordance with the ethical standards of the institutional and/or national research committee(s) and with the Helsinki Declaration (as revised in 2013). Written informed consent was obtained from the patient for publication of this case 
report and accompanying images. A copy of the written consent is available for review by the editorial office of this journal.

Open Access Statement: This is an Open Access article distributed in accordance with the Creative Commons Attribution-NonCommercial-NoDerivs 4.0 International License (CC BY-NC-ND 4.0), which permits the noncommercial replication and distribution of the article with the strict proviso that no changes or edits are made and the original work is properly cited (including links to both the formal publication through the relevant DOI and the license). See: https://creativecommons.org/licenses/by-nc-nd/4.0/.

\section{References}

1. Lansac E, Youssefi P, de Heer F, et al. Aortic Valve Surgery in Nonelderly Patients: Insights Gained From AVIATOR. Semin Thorac Cardiovasc Surg 2019;31:643-9.

2. Etnel JRG, Grashuis P, Huygens SA, et al. The Ross Procedure: A Systematic Review, Meta-Analysis, and Microsimulation. Circ Cardiovasc Qual Outcomes 2018;11:e004748.

3. Falk V, Baumgartner H, Bax JJ, et al. 2017 ESC/EACTS Guidelines for the management of valvular heart disease.

doi: $10.21037 /$ jovs-20-110

Cite this article as: Albertini A, Raviola E, Zucchetta F, Brega C, Mikus E, Tripodi A. Aortic valve neocuspidization with glutaraldehyde-treated autologous pericardium to avoid the prosthesis-patient mismatch of a severely obese 57 -year-old patient—a case report. J Vis Surg 2022;8:24.
Eur J Cardiothorac Surg 2017;52:616-64.

4. Nishimura RA, Otto CM, Bonow RO, et al. 2017 AHA/ ACC Focused Update of the 2014 AHA/ACC Guideline for the Management of Patients With Valvular Heart Disease: A Report of the American College of Cardiology/ American Heart Association Task Force on Clinical Practice Guidelines. Circulation 2017;135:e1159-95.

5. Ozaki S, Kawase I, Yamashita H, et al. Aortic valve reconstruction using autologous pericardium for patients aged less than 60 years. J Thorac Cardiovasc Surg 2014;148:934-8.

6. Etnel JRG, Huygens SA, Grashuis P, et al. Bioprosthetic Aortic Valve Replacement in Nonelderly Adults: A Systematic Review, Meta-Analysis, Microsimulation. Circ Cardiovasc Qual Outcomes 2019;12:e005481.

7. Ross DN. Replacement of aortic and mitral valves with a pulmonary autograft. Lancet 1967;2:956-8.

8. Chan KM, Rahman-Haley S, Mittal TK, et al. Truly stentless autologous pericardial aortic valve replacement: an alternative to standard aortic valve replacement. J Thorac Cardiovasc Surg 2011;141:276-83.

9. Ozaki S. Ozaki Procedure: 1,100 patients with up to 12 years of follow-up. Turk Gogus Kalp Damar Cerrahisi Derg 2019;27:454. 